\title{
La mondialisation des biocarburants et ses limites
}

«Étape intermédiaire pour réduire les émissions de gaz à effet de serre, diversifier les sources d'énergie dans le secteur des transports et préparer l'économie de l'Union européenne à d'autres substituts pas encore prêts pour l'utilisation » ${ }^{1}$, les biocarburants connaissent un engouement généralisé à travers le monde.

Aux États-Unis, $20 \%$ de la production de mais en 2007 devraient être dédiés selon I'USDA à la production d'éthanol, avec lequel I'Energy Policy Act ambitionne de couvrir $30 \%$ de la consommation d'essence en 2030. Et la même évolution est attendue avec le soja pour le biodiesel, avec un doublement cette année des tonnages utilisés.

Premier exportateur et deuxième producteur mondial derrière les USA, le Brésil, avec déjà $70 \%$ de son parc automobile neuf fonctionnant à l'éthanol (moteur flex fuel), accroît ses investissements (73 usines nouvelles s'ajouteront dans les cing prochaines années aux 359 existantes en 2006) et se lance également dans la production de biodiesel.

En Chine, la capacité de production de biocarburants atteindra selon l'administration 12 millions de tonnes en 2020 contre 1,02 million de tonnes aujourd'hui.

Intéressée par ce nouveau marché mondial en phase ascendante, la Malaisie mise sur le biodiesel à partir de l'huile de palme, et s'équipe en conséquence, tandis que les Philippines, la Thaïlande et I'Inde construisent des unités de production d'éthanol.

C'est dans ce contexte mondialisé que l'Union européenne est en passe de devenir le troisième acteur majeur de ce secteur. Dévoilé en début d'année, son plan d'action (orienté majoritairement vers le biodiesel obtenu à partir des huiles végétales) fixe le taux d'incorporation des biocarburants à 5,75 \% en 2010 et à $10 \%$ en 2015 (des objectifs portés en France, par la Loi d'orientation sur l'Énergie à 5,75\% en 2008 et $7 \%$ en 2010). Une perspective qui

\footnotetext{
${ }^{1}$ Stratégie de I'UE en faveur des biocarburants, SEC 2006142.
}

devrait amener la demande européenne en biodiesel (essentiellement composée d'ester de colza) à progresser sur une pente de $20 \%$ par an jusqu'en 2010.

Ces chiffres se traduiront pour la France par la mise en place de six nouveaux sites de production, la création de 15000 emplois et une extension à 2,5 millions d'hectares de la sole totale oléagineuse (contre près de 2 millions aujourd'hui).

Cette «success story » industrielle au niveau mondial de la production des biocarburants dits de première génération, suscite l'enthousiasme légitime de ceux qui en ont porté l'idée durant des années. Mais elle rend aussi d'autant plus vive la nécessité de conformer ces productions aux caractéristiques du contexte général auquel elles se rapportent.

Le premier aspect à prendre en compte tient à la lutte contre le réchauffement climatique. "On ne peut plus raisonner sur une offre qui s'adapterait toujours à une demande en croissance... Aujourd'hui il y a une contrainte absolue, un plafond de gaz à effet de serre qu'il faut tenir durablement dans le temps et à l'échelle de I'humanité ${ }^{2}$ ». Cette contrainte de réduction des émissions des gaz à effet de serre devra s'appliquer en particulier aux conditions de production culturales des matières premières (colza, soja, palme) et en particulier aussi aux conditions de production culturales des huiles importées (4 Mt pour l'Europe à l'horizon 2010). Les textes européens sont clairs sur ce sujet (pour la France, le Cetiom travaille à la mise en place d'une nouvelle Charte pour I'environnement sur la base d'un suivi des bilans énergétiques). Les producteurs d'huile de palme réfléchissent à la définition de critères de durabilité ${ }^{3}$. Mais on peut conjecturer que les

\footnotetext{
${ }^{2}$ Olivier Godard, Rapport au nom de la mission d'information sur l'effet de serre, Assemblée nationale, T2, avril 2006.

${ }^{3}$ OMONT H. Roundtable on Sustainable Palm Oil RSPO. The second RSPO meeting in Jakarta in October 2004. OCL $2005 ; 12(2)$ : 125-8.
}

règles de commercialisation d'âpres débats seront l'objet à tous les niveaux concernés (UE, $\mathrm{OMC}$, etc).

Le deuxième aspect, au moins aussi important, est lié à l'impact des biocarburants sur le fonctionnement des marchés de matières premières agricoles céréales, oléagineux, sucre. Pourra-t-on laisser aux seuls marchés le soin $d^{\prime}$ arbitrer entre les besoins alimentaires et les usages énergétiques et, comme le prévoit ici même M. Von Wissel, laisser le prix de l'huile énergétique déterminer le prix de l'huile alimentaire ? Et si non, quelle instance internationale ou régionale pourrait assurer cette tâche?

Annonçant une ère nouvelle impliquant « un retour important de l'État qui devra fixer les orientations, organiser la coordination, mettre en place les cadres institutionnels adaptés, promouvoir des mécanismes économiques permettant de relayer l'ensemble de ces actions auprès de millions d'agents économiques " ${ }^{4}$...les biocarburants de première génération et leur extension demeurent tributaires de ces questions liées aux dimensions mêmes de leur succès.

Enfin, et pour revenir au niveau plus spécifique corps gras, notons que le biodiesel devrait aussi contribuer dans son sillage à inscrire durablement la chimie verte dans le paysage industriel, et stimuler le développement des bioproduits (biosolvants, biolubrifiants, biotensioactifs, et intermédaires de synthèse sur base oléagineuse) auquel le secteur des huiles et corps gras travaille aussi depuis des années.

Gageons de ce point de vue que "l'or vert» sera tout sauf une "étape intermédiaire ${ }^{5}$.

Jean-Luc GURTLER Jean-Claude ICART

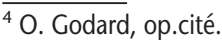

${ }^{5}$ Sur l'ensemble de ces sujets voir ici (dossier) les contributions des intervenants aux Journées Chevreul 2006, organisées par l'Ademe et l'AFECG.
} 